This is the peer reviewed version of the article, which has been published in Acta Paediatrica. 2017, vol. 106, no. 11, pp. 1837-1841. http://dx.doi.org/10.1111/apa.14000. This article may be used for non-commercial purposes in accordance with Wiley Terms and Conditions for Self-Archiving.

\title{
Interleukin 17A gene polymorphism rs2275913 is associated with osteitis after the
}

\section{Bacillus Calmette-Guérin vaccination}

Short title: IL17 gene polymorphism and BCG osteitis

Milla Liehu-Martiskainen ${ }^{1}$, Matti Korppi ${ }^{1}$, Johanna Teräsjärvi ${ }^{2}$, Juho Vuononvirta ${ }^{2}$, Heini Huhtala ${ }^{3}$, Kirsi Nuolivirta ${ }^{4}$, Liisa Kröger ${ }^{5}$, Ville Peltola ${ }^{6}$, Laura Pöyhönen ${ }^{7}$, Qiushui He ${ }^{2,9}$

${ }^{1}$ Center for Child Health Research, Faculty of Medicine and Life Sciences, University of Tampere and University Hospital, Tampere, Finland ${ }^{2}$ Department of Medical Microbiology and Immunology, University of Turku, Turku, Finland

${ }^{3}$ School of Health Sciences, Faculty of Social Sciences, University of Tampere, Tampere, Finland,

${ }^{4}$ Department of Pediatrics, Seinäjoki Central Hospital, Seinäjoki, Finland

${ }^{5}$ Department of Pediatrics, Kuopio University Hospital, Kuopio, Finland,

${ }^{6}$ Department of Pediatrics, University of Turku and University Hospital, Turku, Finland,

${ }^{7}$ St. Giles Laboratory of Human Genetics of Infectious Diseases, Rockefeller Branch,The Rockefeller University, New York, NY, USA,

${ }^{9}$ Department of Medical Microbiology, Capital Medical University, Beijing, China

Corresponding author: Professor Matti Korppi, Center for Child Health Research, Arvo2 building, 33014 University of Tampere, Finland.

Tel +358-50-3186316, Email matti.korppi@uta.fi 
Key words: Bacillus Calmette-Guérin, Innate immunity, Interleukin 17A, Tuberculosis, Vaccination complications

CONFLICTS OF INTEREST

The authors have no conflicts of interest to declare.

FINANCE

Johanna Teräsjärvi, Kirsi Nuolivirta and Laura Pöyhönen received a grant from the Tampere Tuberculosis Foundation to carry out this study. 


\section{ABSTRACT}

Aim: Interleukin-17 (IL-17) appears to promote the host's defense against mycobacterial infections. This study evaluated the association between IL17A gene polymorphism and the risk of Bacillus Calmette-Guérin (BCG) osteitis after newborn vaccination and between IL17A gene polymorphism and IL-17A concentrations in serum.

Methods: IL17A rs2275913 gene polymorphisms and serum IL-17A concentrations were studied in 132 adults aged 21-49 years from across Finland, who had BCG osteitis in infancy after a newborn BCG vaccination. The subjects were recruited in 2007-2008 and their whole blood samples were sent to the National Institute for Health and Welfare, Turku, Finland. Their genotypes and minor allele frequencies were compared with 405 population-based unvaccinated controls aged two to three months from a prospective birth cohort study.

Results: The genotypes and allele frequencies of IL17A rs2275913 differed significantly between the former BCG osteitis patients and controls. The genotype was variant in $75.8 \%$ of cases and $64.0 \%$ of controls $(p=0.012$ ) and the minor allele frequency was $50.0 \%$ in the cases and $41.6 \%$ of the controls $(p=0.009)$. Serum IL-17 concentrations did not differ significantly between the cases with wild or variant genotypes.

Conclusion: IL17A rs2275913 gene polymorphism was associated with a risk of BCG osteitis after vaccination.

\section{Key notes:}

- This study evaluated the association between IL17A gene polymorphism and the risk of Bacillus Calmette-Guérin (BCG) osteitis after newborn vaccination. 
- We studied 132 adults aged 21-49 years from across Finland, who had BCG osteitis in infancy, and 405 population-based unvaccinated controls from a prospective birth cohort study.

- Our findings showed that IL17A rs2275913 gene polymorphism was associated with a risk of $B C G$ osteitis after newborn vaccination.

\section{INTRODUCTION}

Interleukin-17A (IL-17A) is the founding member of the group of pro-inflammatory cytokines produced by T helper 17 (Th17) cells (1). Th17 cells and Th17-derived cytokines participate in the defense against different pathogens, including Mycobacterium tuberculosis (1). The effect of Th17 cells and Th17-derived cytokines may be erroneously directed towards the host and result in various disorders with chronic inflammation, including autoimmune diseases (2).

A systematic review, that included three studies on the role of IL-17A in the Bacillus Calmette-Guerin (BCG) vaccination and six studies on the role of IL-17A in active and latent tuberculosis (TB), showed that the BCG vaccination induced high serum levels of IL17A and high serum interferon-gamma (IFN- $\gamma$ ) levels (3). Both IL-17A and IFN- $\gamma$ increased during the conversion from active to latent TB (3).

In a meta-analysis, IL17F rs763780 and IL17A rs3748067 polymorphisms were associated with susceptibility to TB, but it was significant that this only occurred in Asian populations (4).. However, no significant associations were found between IL17A rs2275913 polymorphism and the risk of TB (4). 
We have previously published our findings on polymorphisms of the mannose binding lectin (MBL) encoding MBL2 gene, the toll-like receptor 2 (TLR2) subfamily encoding genes (TLR1, TLR2, and TLR6) and the IL-10 encoding gene (IL10) in the same cohort of 132 adults. These adults all suffered from BCG osteitis in infancy after newborn BCG vaccinations (5-7). Of the genes that encoded pattern recognising proteins, the variant, low MB production associated genotypes of the MBL2 gene (5), and the wild TLR1 genotype and variant TLR2 and TLR6 genotypes were associated with an increased BCG osteitis risk (6). Interleukins form the next step in innate immunity and four single nucleotide polymorphisms (SNPs) of the IL10 gene had no association with the risk of BCG osteitis (7).

The aim of this study was to evaluate the association between IL17A polymorphism rs2275913 (-197G>A) and the risk of BCG osteitis after newborn vaccination, by comparing the prevalence of that SNP in 132 former BCG osteitis patients and 405 population-based non-vaccinated controls. The genotypes and minor allele frequencies (MAF) were compared between the cases and controls. We also studied the connection between IL17A rs2275913 polymorphism and IL-17 concentrations in serum.

\section{MATERIAL AND METHODS}

\section{Study subjects}

In 2007-2008, we contacted 203 former BCG osteitis patients in Finland with a known current address to ask them if they would take part in this study and $160(78.8 \%)$ replied. They answered a questionnaire that comprised questions on chronic illnesses, long-term 
medication, repeated or chronic infections and mycobacterial infections. Whole blood samples from 132 of the patients, who lived all over Finland, were sent to the laboratory of the National Institute for Health and Welfare, Turku, Finland $(5,8)$. Deoxyribonucleic acid (DNA) was isolated from $200 \mu \mathrm{L}$ of whole blood and both DNA and serum samples were frozen at $-70^{\circ} \mathrm{C}$ for further analyses. For the present study, the frozen samples were transferred to the laboratory of Medical Microbiology and Immunology at the University of Turku, Turku, Finland, where the SNP rs2275913 of the IL17A gene and serum IL-17 concentrations were determined.

BCG osteitis in the study subjects was diagnosed by BCG culture and, or, typical histology from 1960-1988 when the study subjects were infants $(9,10)$. At that time, the diagnostics of BCG osteitis in Finland was centralised at the Public Health Institute in Helsinki. Blood samples for IL17A SNPs and IL-17A concentrations in serum were obtained in 2008-2009 when the subjects were 21 to 49 years old. The study subjects completed a questionnaire and they did not suffer from any acute infections or exacerbations of any chronic disease at the time of sampling (12).

\section{Controls}

The IL17A rs2275913 genotypes were determined in 405 controls recruited from a study called Steps to Children's Healthy Development and Wellbeing, which was a prospective birth cohort study of 1,827 children (12). The control group comprised Finnish infants aged two to three months, who had not yet been vaccinated, apart from the oral rotavirus vaccine. They were all healthy, ethnic Finns, who visited the study clinic from 2008-2010 (13). All the data on the control subjects used in this study, except for their age and gender, were anonymised. 


\section{IL17A rs2275913 genotyping}

All 132 BCG osteitis patients were genotyped for the IL17A rs2275913 gene (-197G>A) by the high-resolution melting analysis (HRMA) (Roche Diagnostics Light Cycler 480, Basel, Switzerland) at the laboratory of the University of Turku (14). The primers (forward 5'TCTGCCCTTCCCATTTTCCTTC-3' and reverse 5'-GGTTAAAATTTCCGCCCCCAATT-3') were designed with Primer-BLAST design tools (National Center for Biotechnology Information, US National Library of Medicine, Maryland, USA). The amplicon size was only 70bp, which was small enough to maximise the difference between melting peaks in variant genotypes and to avoid the other SNPs. Three samples with known genotypes were used to determine the proper concentration of magnesium chloride and annealing temperature for assay. The reaction volume was $20 \mu \mathrm{l}$ for each run and this consisted of $3 \mu \mathrm{l}$ genomic DNA $(\sim 8,3 \mathrm{ng} / \mu \mathrm{l})$ and $17 \mu \mathrm{l}$ of master mix, including $10 \mu \mathrm{l}$ melting master dye and $0.2 \mu \mathrm{M}$ of forward and reverse primers. The master mix provided a final concentration of $3 \mathrm{mM}$ of magnesium chloride. HRMA reactions were run at $95^{\circ} \mathrm{C}$ for $10 \mathrm{~min}$ followed by 45 cycles amplification at $95^{\circ} \mathrm{C}$ for $10 \mathrm{sec}$, at $60^{\circ} \mathrm{C}$ for $10 \mathrm{sec}$ and at $72^{\circ} \mathrm{C}$ for $15 \mathrm{sec}$. After the polymerase chain reaction process, the final melting cycle conditions were as outlined by Roche: heating to $95^{\circ} \mathrm{C}$ and holding for one minute, cooling to pre-hold temperature at $40^{\circ} \mathrm{C}$ and following this with a melting interval to collect fluorescence from $60^{\circ} \mathrm{C}$ to $95^{\circ} \mathrm{C}$, at a ramp rate of $0.2^{\circ} \mathrm{C}$ per second. In each run, known IL17A rs2275913 standards, namely wild type, heterozygote and homozygote variants, were used.

All the 405 controls were genotyped for IL17A rs2275913 (-197G>A) polymorphism. Genotyping from extracted DNA was performed by the Sequenom MassARRAY iPlex Gold 
system (Sequenom Inc, California, USA) at the University of Eastern Finland, Kuopio, Finland, as previously described (13).

The functionality of the IL17A rs2275913 (-197G>A) polymorphism has been documented in cell cultures obtained from healthy subjects (15). In vitro stimulated T cells from those possessing the variant $A$ allele produced significantly more IL-17 than those without the $A$ allele.

\section{Serum IL-17A measurement}

Serum samples were available from 127 of the 132 former BCG osteitis patients at 21-49 years of age and this meant that their IL-17A concentrations could be measured. The BioPlex Pro Human IL-17A/F immunoassay kit (Bio-Rad, Helsinki, Finland) was used to measure serum IL-17A. The measurement run was carried out using the Bio-Plex 200 System (Bio-Rad, Helsinki, Finland) and IL-17A measurements were performed according to manufacturer's protocol. One sample with known concentration of IL-17A was used as an internal control in all runs. The detection limit of IL-17A was $1.22 \mathrm{pg} / \mathrm{ml}$ (13).

\section{Statistical analyses}

The Statistical Package for the Social Sciences Statistics for Windows, version 19 (IBM Corp, New York, USA) was used for the statistical analyses. The distributions of the genotypes and MAFs between cases and controls were compared using chi-square and Fisher's exact tests, as appropriate. Exploratory data analyses revealed that serum IL-17 concentrations were non-normally distributed. Therefore, the Mann-Whitney U test was used to compare the concentrations as continuous variables and Fisher's exact test was 
used for categorised variables between the genotypes. The results are given as percentages, medians, interquartile (IQ) ranges $25 \%-75 \%$ and minimum to maximum ranges.

\section{Ethics}

The study was approved by the Ethics Committee of the Tampere University Hospital District and written consent was obtained from the study subjects, including permission to perform genetic studies concerning susceptibility to mycobacterial infections. The samples studied in the laboratory were coded so that any personal identification data was removed. The study protocol for the control children was approved by the Ethics Committee of the Hospital District of South-West, Turku, Finland and written consent was obtained from the parents to use their children's blood samples.

\section{RESULTS}

The genotypes of IL17A rs2275913 polymorphism were analysed in 132 former BCG osteitis patients with slightly more females ( $n=73,55.3 \%)$ than males. At the time of sampling, $21(15.9 \%)$ of the subjects were $21-30$ years old, $88(66.7 \%)$ were $31-40$ years old and 21 (15.9\%) were 41-50 years old.

The genotypes of IL17A rs2275913 (-197G>A), as well as the MAFs, differed significantly between the 132 former BCG osteitis patients and 405 population controls (Table 1). The minor allele A was more common in the cases $(50.0 \%)$ than the controls $(41.6 \%)$, as were 
the variant genotypes GA (50.0\% versus $44.7 \%)$ and $A A(25.8 \%$ versus $19.1 \%)$ and the combined variant genotypes $\mathrm{GA}$ and $\mathrm{AA}(75.8 \%$ versus $64.0 \%)$.

The median concentration of serum IL-17A in the 127 former BCG osteitis patients was $27.09 \mathrm{pg} / \mathrm{ml}$ (IQ 13.68-62.25, range 0.00-1973.52). The median IL-17A concentration was $22.68 \mathrm{pg} / \mathrm{ml}$ in 71 females and $32.56 \mathrm{pg} / \mathrm{ml}$ in 59 males $(\mathrm{p}=0.257)$. At the time of sampling, the median IL-17A concentration was $35.56 \mathrm{pg} / \mathrm{ml}$ in those aged 21-30 years, $28.94 \mathrm{pg} / \mathrm{ml}$ in those aged $31-40$ years and $22.68 \mathrm{pg} / \mathrm{ml}$ in those aged $41-50$ years $(p=0.949)$.

There were no significant differences in IL-17A concentrations between those with wild and variant genotypes of IL 17A rs2275913. As seen in Figure 1, the distributions were wide and there were three outliers and eight extreme outliers. The result was negative when IL-17A concentrations were analysed as continuous variables (Figure 1) and as categorised variables with the limits of $25^{\text {th }}, 50^{\text {th }}$, and $75^{\text {th }}$ percentiles, or as detected versus non-detected (data not shown).

\section{DISCUSSION}

Two main results emerged from this controlled study on IL17A rs2275913 (-197G>A) polymorphism in BCG osteitis. First, the GA and AA variant genotypes were significantly more common in former BCG osteitis patients than in population-based controls and, in line with this, the minor allele A frequencies were significantly higher in BCG osteitis cases than in controls. Second, the serum concentrations of IL-17A, in the former BCG osteitis patients in adulthood, did not differ significantly between those with variant or wild genotypes. 
The result that the SNP rs2275913 of the IL17A gene differed significantly between the former BCG osteitis patients and controls, suggests that IL-17A plays a role in the development of BCG osteitis after a BCG vaccination. No previous data are available on the association of IL17A gene polymorphisms with response to $B C G$ vaccinations or with vaccination complications like BCG osteitis. Some data have been published on different IL17 polymorphisms and tuberculosis, as summarised in a systematic review (4). IL17A rs3748067 and IL17F rs763780 polymorphisms were associated with susceptibility to tuberculosis in Asian populations, but not in European populations. However, there were no significant associations between the IL17A rs2275913 polymorphism we examined in the present study and the tuberculosis risk in either Asian or European populations (4). IL17A rs2275913 polymorphism has been associated with a decreased tuberculosis risk in the Brazilian population (16).

Serum IL-17A concentrations did not differ between those with variant or wild genotypes and this result was similar in continuous analyses and in categorised analyses that focused on low concentrations. Our observations differed from the findings of a Finnish study that measured serum IL-17A in 93 of the 405 controls included in the present study at 13 months of age. That study found that IL-17A was detectable in $6 \%$ of the variant AA homozygotes, in $33 \%$ of the variant GA heterozygotes and in $75 \%$ of the wild GG homozygotes (13). The concentrations were much higher in the adults in the present study: the median was $26.3 \mathrm{pg} / \mathrm{ml}$ in those with the variant genotype and $28.94 \mathrm{pg} / \mathrm{ml}$ in those with the wild genotype, compared to $<1.25 \mathrm{pg} / \mathrm{ml}$ and $2.5 \mathrm{pg} / \mathrm{ml}$ in the infant controls, respectively (13). The measurements were carried out with the same method in the same laboratory, which means that the differences were not technical. 
A systematic review published in 2012 (3), included three studies carried out in infants and children vaccinated with BCG (17-19). Serum IL-17 concentrations increased significantly, as did IFN-y concentrations, when they were measured three to 12 months after the BCG vaccination, compared to pre-vaccination concentrations. There are three possible explanations for our negative result on the association between IL17A rs2275913 polymorphism and serum IL-17A concentrations. An increase in the cytokine concentrations may need a stimulus, such as an ongoing infection or a recent vaccination, which was no longer present in our adult cohort. The rs2275913 polymorphism we selected does not influence the production of IL-17A, although this polymorphism has been shown to be functional in cell cultures obtained from healthy subjects (15). The third, theoretical possibility is that BCG osteitis in early childhood programmed innate immunity to respond with high IL-17 production to different stimuli independently from the IL17 genotype. In the present study, the concentrations were multiplied many times when they were compared to those measured in the 13-month-old healthy non-vaccinated children by the same method in the same laboratory (14).

The main strength of the present study was the unique study cohort of more than 130 patients who presented with firmly diagnosed BCG osteitis after receiving the BCG vaccination as newborn infants. Originally, the total number of BCG osteitis patients was 222, and as previously published, none of them had died of tuberculosis, the BCG infection or another severe infection $(9,11)$. The blood samples were taken when the study subjects were adults. The fact that samples were not collected during the BCG infection cannot influence the distribution of the polymorphisms, but may explain the absence of any significant associations between serum IL-17A concentrations and the subjects with wild 
and variant genotypes. The other strength of the study was that it was carefully controlled with regard to polymorphisms, by using more than 400 population-based controls. Despite this, the material is rather small for genetic studies, and a risk of type-2 statistical errors may be present. In addition, only one IL17A polymorphisms was studied, but just this rs2275913 is best studied among different IL17A polymorphisms, and there is both in vitro and in vivo evidence that it is functional regulating IL-17A production.

\section{CONCLUSION}

We found evidence that the IL17A rs2275913 (-197G>A) gene polymorphism was associated with the risk of BCG osteitis after BCG vaccination. The variant genotypes and minor alleles were significantly more common in the 132 former BCG osteitis patients studied as adults than in 405 population-based controls studied in early infancy. Since BCG vaccination is used globally to protect children from tuberculosis, and risk groups are still vaccinated in western countries, research is needed to find genetic or immunologic markers to identify children at risk of complications after vaccination.

\section{ABBREVIATIONS}

BCG, Bacillus Calmette Guérin

DNA, deoxyribonucleic acid

HRMA, high-resolution melting analysis

IFN- $\gamma$, interferon gamma

IL 10, interleukin 10

IL 17, Interleukin 17

MAF, minor allele frequency

TB, tuberculosis 


\section{REFERENCES}

1. Torrado E, Cooper AM. IL-17 and Th17 cells in tuberculosis. Cytokine Growth Factor Rev 2010; 21: 455-62.

2. Isailovic N, Daigo K, Mantovani A, Selmi C. Interleukin-17 and innate immunity in infections and chronic inflammation. $J$ Autoimmun 2015; 60: 1-11.

3. Li Q, Li J, Tian J, Zhu B, Zhang Y, Yang K, et al. IL-17 and IFN-y production in peripheral blood following BCG vaccination and Mycobacterium tuberculosis infection in human. Eur Rev Med Pharmacol Sci 2012;16: 2029-36.

4. Zhao J, Wen C, Li M. Association analysis of Interleukin-17 gene polymorphisms with the risk susceptibility to tuberculosis. Lung 2016; 194: 459-67.

5. Pöyhönen L, Kröger L, Gröndahl-Yli-Hannuksela K, Vuononvirta J, Huhtala H, He Q, et al. Variant MBL2 genotypes producing low mannose-binding lectin may increase risk of Bacillus Calmette-Guerin osteitis in vaccinated newborns. Acta Paediatr 2013; 102: 1095-9.

6. Pöyhönen L, Nuolivirta K, Vuononvirta J, Kröger L, Huhtala H, Mertsola J, et al. Toll-like receptor 2 subfamily gene polymorphisms are associated with Bacillus Calmette-Guérin osteitis following newborn vaccination. Acta Paediatr 2015; 104: 485-90. 
7. Pöyhönen L, Teräsjärvi J, Nuolivirta K, Vuononvirta J, Gröndahl-Yli-Hannuksela K, Kröger L, et al. Interleukin-10 gene promoter region polymorphisms are not associated with BCG osteitis in vaccinated infants. Int J Tuberc Lung Dis 2015; 19: 1158-62.

8. Pöyhönen L, Kröger L, Huhtala H, Mäkinen J, Mertsola J, Martinez-Barricarte R, et al. Interferon-gamma-dependent Immunity in Bacillus Calmette-Guérin vaccine osteitis survivors. Pediatr Infect Dis J 2016; 35: 690-4.

9. Kröger L, Brander E, Korppi M, Wasz-Höckert O, Backman A, Kröger H, et al. Osteitis after newborn vaccination with three different Bacillus Calmette-Guérin vaccines: twenty-nine years of experience. Pediatr Infect Dis J 1994; 13: 113-6.

10. Kröger L, Korppi M, Brander E, Kröger H, Wasz-Höckert O, Backman A, et al. et al. Osteitis caused by Bacille Calmette-Guérin vaccination: a retrospective analysis of 222 cases. J Infect Dis 1995; 172: 574-6.

11. Pöyhönen L, Pauniaho SL, Kröger L, Korppi M. Orthopedic complications in former Bacillus Calmette-Guérin osteitis patients. Pediatr Infect Dis J 2016; 35: 579-80 12. Lagström H, Rautava $P$, Kaljonen A, Räihä H, Pihlaja P, Korpilahti $P$, et al. Cohort profile: Steps to the healthy development and well-being of children (the STEPS study). Int J Epidemiol 2013; 42: 1273-1284.

13. Vuononvirta J, Peltola V, Ilonen J, Mertsola J, He Q. The gene polymorphism of IL$17 \mathrm{G} 152 \mathrm{~A}$ is associated with increased colonization of Streptococcus pneumoniae in young Finnish children. Pediatr Infect Dis J 2015; 34: 928-32.

14. Teräsjärvi J, Hakanen A, Korppi M, Nuolivirta K, Gröndahl-Yli-Hannuksela K, Mertsola J, et al. Rapid detection of functional gene polymorphisms of TLRs and IL-17 using high resolution melting analysis. Sci Rep 2017 Feb 2; 7: 41522. 
15. Espinoza JL, Takami A, Nakata K, Onizuka M, Kawase T, Akiyama H, et al. A genetic variant in the IL-17 promoter is functionally associated with acute graftversus-host disease after unrelated bone marrow transplantation. PLoS One 2011; 6: e26229.

16. Milano M, Moraes MO, Rodenbusch R, Carvalho CX, Delcroix M, Mousquer G, et al. Single Nucleotide Polymorphisms in IL17A and IL6 Are Associated with Decreased Risk for Pulmonary Tuberculosis in Southern Brazilian Population. PLoS One 2016; 11: e0147814.

17. Lalor MK, Smith SG, Floyd S, Gorak-Stolinska P, Weir RE, Blitz R, et al. Complex cytokine profiles induced by BCG vaccination in UK infants. Vaccine 2010; 28 : $1635-41$

18. Burl S, Adetifa UJ, Cox M, Touray E, Ota MO, Marchant A, et al. Delaying bacillus Calmette-Guérin vaccination from birth to $41 / 2$ months of age reduces postvaccination Th1 and IL-17 responses but leads to comparable mycobacterial responses at 9 months of age. J Immunol 2010; 185: 2620-8.

19. Smith SG, Lalor MK, Gorak-Stolinska P, Blitz R, Beveridge NE, Worth A, et al. Mycobacterium tuberculosis PPD-induced immune biomarkers measurable in vitro following BCG vaccination of UK adolescents by multiplex bead array and intracellular cytokine staining. BMC Immunol 2010; 11: 35. 
Table 1. Genotypes of IL17A rs2275913 polymorphisms and minor allele A frequencies in 132 former BCG osteitis patients and 405 population controls.

\begin{tabular}{|c|c|c|c|c|c|}
\hline \multirow[t]{2}{*}{ Genotypes } & \multicolumn{2}{|c|}{$\begin{array}{l}\text { BCG osteitis } \\
\text { group } \\
\mathrm{n}=132\end{array}$} & \multicolumn{2}{|c|}{$\begin{array}{l}\text { Control group } \\
\mathrm{n}=405\end{array}$} & \multirow{2}{*}{$\begin{array}{l}\text { P value } \\
\text { between } \\
\text { cases and } \\
\text { controls }\end{array}$} \\
\hline & $n$ & $\%$ & $n$ & $\%$ & \\
\hline GG, wild & 32 & 24.2 & 146 & 36.0 & --- \\
\hline GA, variant & 66 & 50.0 & 181 & 44.7 & $0.035^{\#}$ \\
\hline $\mathrm{AA}$, variant & 34 & 25.8 & 78 & 19.1 & $0.014^{\#}$ \\
\hline $\begin{array}{l}\text { Variant all, GA or } \\
\text { AA }\end{array}$ & 100 & 75.8 & 259 & 64.0 & $0.012^{\#}$ \\
\hline Allele A (minor) & $132 / 264$ & 50.0 & $337 / 810$ & 41.6 & 0.009 \\
\hline
\end{tabular}

Distribution of three genotypes between BCG cases and controls: $p=0.033$ between cases and controls

\# Compared to the wild type 
Figure 1. Serum IL-17A concentrations in 127 former BCG osteitis patients in relation to wild and variant genotypes of the IL17A rs2275913 polymorphism.

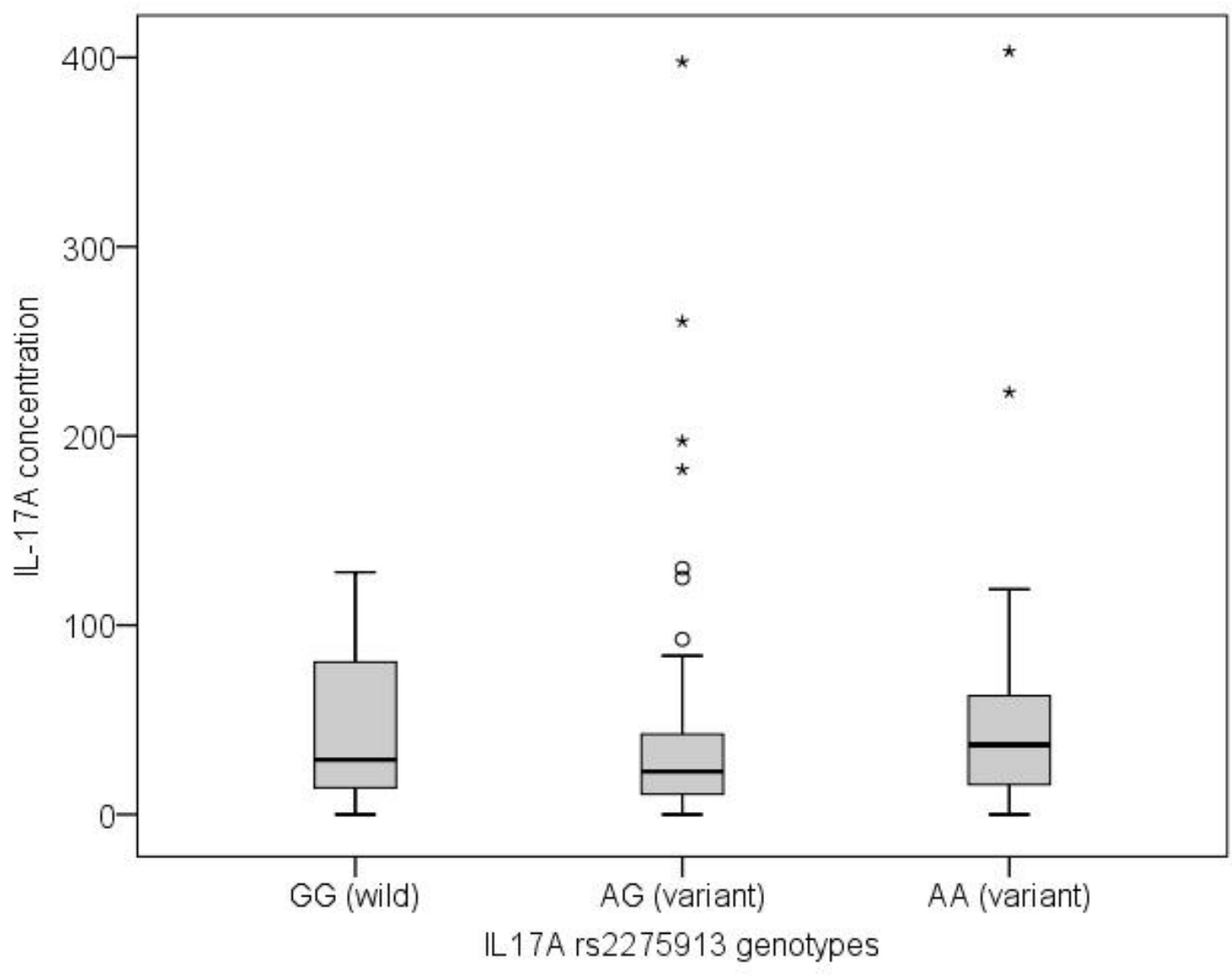

The box-plot figure expresses medians and quartiles (Q1, Q3), outliers (Q3 $+1.5 x$ interquartile range Q3-Q1) expressed as small circles $^{\circ}$ and extreme outliers (Q3 $+2.0 x$ $1.5 \mathrm{x}$ interquartile range) expressed as stars*. Two extreme outliers $(1973.52 \mathrm{pg} / \mathrm{ml}$ in the GG group and $1316 \mathrm{pg} / \mathrm{ml}$ in the GA group) are situated outside the figure. 